\title{
Can experiments in nonhuman primates expedite the translation of treatments for spinal cord injury in humans?
}

\author{
Grégoire Courtine ${ }^{1}$, Mary Bartlett Bunge ${ }^{2}$, James W Fawcett ${ }^{3}$, Robert G Grossman ${ }^{4}$, Jon H Kaas ${ }^{5}$, Roger Lemon ${ }^{6}$, \\ Irin Maier ${ }^{7}$, John Martin ${ }^{8}$, Randolph J Nudo ${ }^{9}$, Almudena Ramon-Cueto ${ }^{10}$, Eric M Rouiller ${ }^{11}$, Lisa Schnell ${ }^{9}$, \\ Thierry Wannier ${ }^{11}$, Martin E Schwab ${ }^{9} \&$ V Reggie Edgerton ${ }^{1}$
}

Progress continues in the development of reparative interventions to enhance recovery after experimental spinal cord injury (SCI). Here we discuss to what extent rodent models of SCI have limitations for ensuring the efficacy and safety of treatments for humans, and under what circumstances it would be advantageous

${ }^{1}$ Department of Physiological Science and

Neurobiology, and Brain Research Institute, University of California, Los Angeles, California 90095, USA. ${ }^{2}$ The Miami Project to Cure Paralysis, Department of Cell Biology and Anatomy, and Department of Neurological Surgery, University of Miami School of Medicine, Miami, Florida 33101, USA. ${ }^{3}$ Brain Repair Center, University of Cambridge, Cambridge CB2 2PY, UK. ${ }^{4}$ Neurological Institute, Department of Neurosurgery, The Methodist Hospital, Houston, Texas 77030, USA. ${ }^{5}$ Department of Psychology, Vanderbilt University, Nashville, Tennessee 37203, USA. ${ }^{6}$ Institute of Neurology, University College London, London WC1N 3BG, UK. ${ }^{7}$ Brain Research Institute, University of Zurich, Zurich $\mathrm{CH}-8057$, Switzerland. ${ }^{8}$ Center for Neurobiology and Behavior, Columbia University, New York, New York 10032, USA. ${ }^{9}$ Landon Center on Aging, Department of Molecular and Integrative Physiology, Kansas University Medical Center, Kansas City, Kansas 66160, USA. ${ }^{10}$ Laboratory of Neural Regeneration, Institute of Biomedicine, Spanish Council for Scientific Research, Valencia 46010, Spain. ${ }^{11}$ Institute of Physiology, University of Fribourg, Fribourg $\mathrm{CH}-1700$, Switzerland. Correspondence should be addressed to V.R.E. (vre@ucla.edu).

Published online 3 May 2007; doi:10.1038/nm1595 or necessary to test treatments in nonhuman primates before clinical trials. We discuss crucial differences in the organization of the motor systems and behaviors among rodents, nonhuman primates and humans, and argue that studies in nonhuman primates are critical for the translation of some potential interventions to treat $\mathrm{SCI}$ in humans.

Traumatic SCI has long-term health, economic and social consequences worldwide ${ }^{1,2}$, giving a sense of the urgency to the development of ways to treat it. Treatments that lead to at least partial functional recovery after SCI can substantially improve the quality of life of affected individuals. Consequently, there is considerable need to take to the clinic those interventions that have shown effectiveness in promoting functional improvement in laboratory animals.

Progress continues in the identification of interventions that augment plasticity after injury by promoting axonal regeneration and sprouting in rodents ${ }^{3-6}$. Some of these treatments may be efficacious in patients with SCI, and have or are entering phase 1 clinical trials. Important differences exist, however, between the nervous systems of rodents and humans, in terms of size, neuroanatomical, neurophysiological and behavioral characteristics, and inflammatory and immunological responses. Here we point out limitations of the commonly used rodent models in ensuring the efficacy and safety of SCI treatments for humans, and discuss how the use of nonhuman primates can facilitate the successful advancement of potential treatments to clinical trials.

We primarily focus on motor performance, as this behavior is well studied and there are important similarities between some nonhuman primates and humans in the organization of the neural systems that control movement. However, a similar examination of autonomic and other neural functions compromised after SCI would also be useful for a more comprehensive strategic approach in optimizing functional recovery after SCI (Box 1). We conclude that studies in nonhuman primates can probe the effects of therapy-induced neural plasticity on multiple aspects of functional recovery with a refinement that cannot be attained in rodents.

\section{Important differences between rodents and primates}

Neuroanatomy. Although there is remarkable conservation among the motor systems of vertebrates, some features have undergone pronounced evolutionary changes ${ }^{7,8}$. This is particularly true of the motor cortex and its descending output - the corticospinal tract (CST) — which projects extensively to the brainstem and spinal cord in primates. In many primates, the CST can influence motoneuron activity both directly and indirectly ${ }^{8}$. Indeed, over the course of the evolution of human and nonhuman primates there has been a massive increase in the proportion and actual size of the neocortex that gives rise to the CST, a fast-conducting component of the CST has appeared, and the corticospinal axons have changed location from the dorsal to the lateral columns of the spinal cord 9 .

Differences between rodents and primates in the pattern of CST terminations are qualitative and quantitative. In rodents, the CST projects mainly to dorsal horn neurons and premotor 


\section{COMMENTARY}

spinal circuits. In many nonhuman primates, such as the rhesus monkey, the projection pattern of the CST is much more complex: a significant proportion of CST fibers projects to the ventral horn, and some axons synapse directly on motoneurons ${ }^{8}$, in particular those innervating hand muscles. In humans, this trend is even more marked ${ }^{10}$. Stimulation of CST neurons in the motor cortex evokes motor responses that markedly differ in primates compared to rodents ${ }^{11}$, as well as between different primate species ${ }^{8}$. For example, there is a strong correlation between the number of direct connections between cortex and motor neurons and the level of manual dexterity of nonhuman primate species (ref. 8 and Fig. 1).

There also are substantial differences between rodent and most primates in the distances over which neural fibers might be required to regenerate after injury. This difference could limit the inferences that can be made from regeneration studies between rodents and primates. This is relevant for injury to the cervical spinal cord, but may be even more problematic for reinnervation of the lumbar regions, owing to the long distance that fibers may need to travel to reach locomotor circuits in humans.

Behavior. The development of the descending motor pathways has enabled primates to perform incrementally recruit portions of the motoneuron pools that innervate distal mus-

\section{Box 1 There are critical differences in other neurological systems of rodents, nonhuman primates and humans}

Although our focus is on neuromotor function and the safety of interventions designed to improve function after $\mathrm{SCl}$, other functional aspects should be similarly examined with respect to the potential advantages of using nonhuman primates instead of rodents. For example, methods to improve recovery of autonomic functions are ranked as very important by $\mathrm{SCl}$ patients ${ }^{1}$. The overall architecture of the autonomic neural pathways is similar among mammals, but bladder control and sexual function are more similar between humans and nonhuman primates than between humans and rodents. Issues of autonomic control, such as blood pressure changes when assuming a vertical posture after a $\mathrm{SCl}$, are also important, and nonhuman primate experiments are likely to provide a better predictor of the effects of a given treatment on humans than findings from rodents.

cles, and has contributed to an increased ability to control hand musculature ${ }^{11-13}$. In particular, there is evidence that the appearance of direct cortical projections to spinal motoneurons correlates with the emergence of precision grip between the thumb and the index fingers, which exists in only some primates (ref. 8 and Fig. 1). Accordingly, and unlike in rodents ${ }^{13-15}$, interruption of the cortical projections to the spinal cord in primates causes a major impairment in fine motor function of the hands and feet, the magnitude of which is most severe in humans (ref. 16 and Table 1).

Likewise, CST lesions have little effect on stepping in rodents ${ }^{17}$, indicating that the motor cortex is not essential for creating the muscle synergies that sustain simple locomotion in rats and mice. By contrast, damage to the CST in rhesus monkeys provokes permanent deficits during stepping ${ }^{15,18}$, and in humans, CST damage leads to a motor impairment severe enough to compromise independent walking ${ }^{16}$.

So, even though fine motor control of the forelimb can be tested in rodents and is affected by lesions to the CST (ref. 19), the finesse in digital control is far less developed in rodents than in nonhuman primates, and there are marked differences in the musculoskeletal design of the forelimb, hand and distal digit musculature between primates and rodents. Although it has not been directly explored,
Rodents (rats)

Manual

dexterity

Amplitude of corticomotoneural-EPSPs
New world monkeys (squirrel)

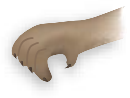

5
Old world monkeys (macaque)

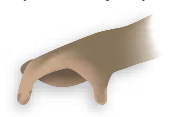

6
Humans

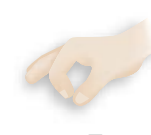

7 $2 \mathrm{mV}$

Number of fibers $\quad 137,000$ per tract

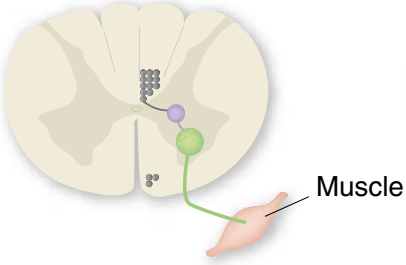

Interneuron

Motoneuron

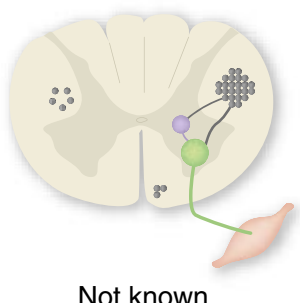

Not known

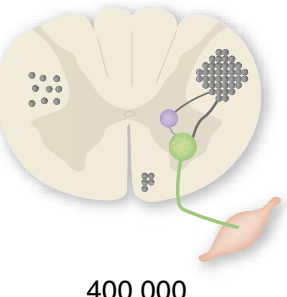

400,000

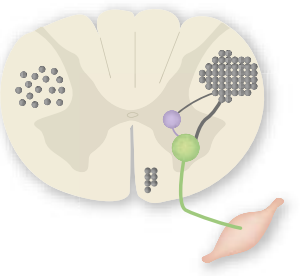

$1,101,000$

Figure 1 Relationship between the development of the corticospinal tract and the emergence of fine motor control abilities. In rodents, there are no direct connections between corticospinal neurons and the cervical motoneurons that innervate forelimb muscles-interneurons relay cortical input to motor neurons. In the evolution of the corticospinal tract in nonhuman primates and humans, direct corticospinal connections with motoneurons have emerged, together with an increase in the size and number of the corticospinal fibers. Accordingly, the size of the excitatory postsynaptic potential (EPSP) elicited by cortical neurons on motoneurons has increased during primate evolution. Furthermore, most of the corticospinal tract fibers in rodents travel in the dorsal columns. In contrast, the primate corticospinal tract is mostly located in the lateral columns, and a significant proportion of corticospinal fibers (10-20\%) descend ipsilaterally. Development of the corticospinal tract correlates with the improvement in the index of dexterity (as quantified in ref. 30), particularly in the ability to perform finger-thumb precision grip. Figure adapted from ref. 31. 
Table 1 Effect of interruption of corticospinal tract on locomotion and manual dexterity

\begin{tabular}{lll}
\hline & \multicolumn{1}{c}{ Locomotion } & \multicolumn{1}{c}{ Manual dexterity } \\
\cline { 2 - 3 } Rodents & $\begin{array}{l}\text { No significant disruption of the } \\
\text { locomotor function }\end{array}$ & $\begin{array}{l}\text { Significant alteration followed by } \\
\text { limited to extensive recovery }\end{array}$ \\
Macaque monkeys & Some permanent locomotor deficits & $\begin{array}{l}\text { Complete loss of function followed by } \\
\text { limited recovery }\end{array}$ \\
Humans & Loss of independent walking capabilities & Permanent deficits of function \\
\hline
\end{tabular}

testing in nonhuman primates might therefore provide a better prediction of the potential of therapies for SCI to mediate recovery of manual dexterity and stepping.

Detailed assessments of motor capacity can be comprehensive in nonhuman primate SCI studies. From the perspective of clinical trials, an advantage of testing motor performance in nonhuman primates is the similarity in functional measures between them and humans, as compared to rats. Indeed, the precision grip, pre-shaping of the hand, grasping and other manual prehensile tasks performed by macaques and other Old World monkeys are very similar to these behaviors in humans. By contrast, testing fine motor control in rodents remains limited to a coarse success-rate assessment. In rare cases, time-consuming video analysis has been used, but the detailed fine control of the distal phalanges in rodents remains rudimentary ${ }^{19}$. Moreover, assessment of cortical connectivity, supraspinal access to spinal motoneurons and segmental circuit properties can be performed similarly in nonhuman primates and humans.

Evaluation of motor behavior after SCI should include careful documentation of how different manual functions are performed over the course of the recovery period. In particular, combined analyses of motor pool recruitment patterns and kinematics of the head, trunk and limbs can provide decisive information on the degree to which the animal recovers using compensatory strategies to perform the task successfully. Transcranial magnetic stimulation, magnetic resonance imaging and measurement of sensory-evoked potentials can provide additional tools for obtaining very similar motor performancerelated data in nonhuman primates and humans.

It is also important to assess stepping ability and other key motor functions. The extent to which stepping and grasping differ in their underlying neural processes and the primate's intrinsic capacity to recover these functions after a SCI are not fully understood ${ }^{15}$. Engaging the neural circuits for stepping may facilitate the recovery of timing in the recruitment of antagonistic motor pools after injury and promote restoration of fine motor control (G. Courtine et al., Soc. Neurosci. Abstr. 654.7,
2004), and this capability may be unique to nonhuman primates.

In examining locomotor behavior, it is also possible to study bipedal walking in primates. In addition to quadrupedal locomotion, many primate species practice bipedal walking with characteristics close to human walking, and bipedal stepping on a treadmill or over ground can be tested and quantified ${ }^{20}$. Furthermore, manually or robotically assisted bipedal step training after SCI can be implemented in nonhuman primates.

Beyond the CST. Differences in motor systems suggest that strategies to promote regeneration of the nervous system might influence rodents differently from primates, resulting in divergent anatomical and functional outcomes. Even if there were similar outcomes, the neurological basis for the improvement may differ substantially, owing to these functional differences. For example, compared to rodents, primates engage more complex neural circuits in the parietal and frontal lobes of the cerebral cortex even for the simplest of skilled movements.

This reliance of primates on the cortex for motor function may, in turn, offer greater plasticity and recovery after partial SCI. For example, sprouting of spared CST fibers to the descending motor systems and associated changes in the organization of the cortex may provide a unique capacity for plasticity, which can lead to improvement in motor function, particularly if fiber growth can be enhanced. Treatments that promote plasticity in rodents might be considerably more efficacious in nonhuman primates and in humans. So, although technically challenging, there is a clear need to analyze changes in the cerebral cortex ${ }^{18,21-23}$ as well as responses of non-CST descending pathways when testing a treatment in primates with SCI. The potential for reorganization at multiple sites in the brain after SCI may be an effective means to enhance motor recovery in response to incomplete injuries ${ }^{24}$.

Similarly, to what extent could regeneration of a few ascending fibers across the injury site restore sensory function and contribute to improved motor behavior? These possibilities could not be tested with the same resolution in rodents, as sensory discrimination does not seem to be as critical for manual dexter- ity in rodents as it is in primates, nor is the response to injury of the sensory area in the brain similar ${ }^{25}$.

\section{When should we choose nonhuman primates over rodents?}

Rodent and feline models have been used for the development of the current treatments that are under consideration for use after a SCI, and they have to remain the mainstay of experimentation. However, testing some forms of treatments in nonhuman primates before undertaking human trials is likely to provide essential information on the efficacy as well as the possible adverse effects of specific treatments. As implied above, a major advantage in using nonhuman primates is the technical capability to comprehensively examine a range of highly skilled motor functions of the hand using electrophysiological and biomechanical tools. Furthermore, it is feasible to monitor the degree to which, and how, different neuromuscular components contribute to the wide range of hand functions. There are, however, other factors that should inform our decision to use nonhuman primates to test therapies for SCI.

Efficacy and safety. The key factor in deciding whether a potential intervention should be studied in nonhuman primates instead of rodents relates to efficacy and safety: to what extent can the efficacy and safety of a given treatment be assessed in rodent studies? There are many questions that can be addressed effectively in the rodent that can improve the probability of successful extrapolation to humans.

In the context of cell-replacement therapy, for example, there are several questions for which studies in rodents can be very informative: which cell types and how many cells should be implanted, what percentage of cells survive after implantation, what the postoperative procedures should be to maximize their effectiveness, how much of a specific growth factor should be administered, and what types of immunological, urological, respiratory, sensory and motor effects might be expected to occur, and over what time frame.

In addition, studies in rodents can give us early warnings of the secondary effects of a given therapy. For example, aberrant axonal sprouting associated with allodynia-like hypersensitivity of the forepaws has been reported after intraspinal graft of neural stem cells in a model of rodent SCI (ref. 26).

Although work in rodents has answered some of these primary questions, studies in nonhuman primates might be necessary to more accurately predict the optimal treatment procedures to use in humans. In the same context of cell-replacement therapy, or when studying the administration of growth 


\section{Box 2 Which nonhuman primate should we use?}

The complexity and refinement in the organization of the cortical and spinal circuitry underlying motor behavior have increased gradually during primate evolution from New World and Old World monkeys to apes and humans. Accordingly, the nonhuman primate to be used will depend on the specific questions being asked. However, on the basis of current experience and neurophysiological and neuroanatomical data, the macaque monkey provides a clear advantage for the translation of the findings to humans. Owing to its relatively large size, the macaque monkey is more comparable to humans with respect to metabolism, pharmacodynamics, time frame of treatment and blood-brain barrier properties. Moreover, the macaque monkey, as opposed to marmosets, squirrels and spider monkeys, has the advantage of being easily trained to use the hand, presumably because the projection patterns of its CST (including direct connections with motoneurons) and its nonprimary motor cortical areas are most similar to those of humans. Macaque monkeys also possess the cognitive ability to learn and to perform tasks used by humans. Indeed, they have historically been the monkeys of choice for all studies involving single-unit recordings during fine motor control movements, as well as for research on brain-machine interfaces. Consequently, macaque monkeys are better suited than prosimian primates and New World monkeys to model humans when the potential of neural repair interventions that promote recovery of fine motor skills after a $\mathrm{SCl}$ or other debilitating condition is being investigated ${ }^{8}$. In fact, there is currently no good scientific rationale for carrying out $\mathrm{SCl}$ research in apes (bonobo, chimpanzee, gorilla and orangutan).

factors or agents that neutralize neurite growth inhibitors, experiments in nonhuman primates using the conditions outlined by experiments in rodents could help insure faster, safer and more efficacious use in humans with SCI (ref. 14).

Likewise, inflammatory and immune responses differ between primates and rodents ${ }^{7}$, and could contribute to altered secondary cell damage, removal of debris after trauma, neural plasticity and functional recovery. These differences could render a neural intervention that is beneficial in rodents to be ineffective in primates. These factors suggest that potential interventions can be tested stringently in nonhuman primates while providing quantitative assessment of efficacy. In effect, these results could critically enhance the safety of the patients participating in a clinical trial.

Ethical and financial considerations. There are continuing discussions regarding the instances in which nonhuman primates can be used in research. For example, in a recently published report ${ }^{27}$, the UK's Medical Research Council and the Wellcome Trust concluded that, although there is a need to review the ethical and scientific justification for primate use and for strict legal controls, some biomedical problems are such that alternatives are not available or appropriate and it is important to conduct research on nonhuman primates. In the case of SCI, trying to optimize the conditions for a given treatment in human studies would be limited by the number of patients available for trials and would be highly problematic from an ethical standpoint.
Admittedly, almost all studies on nonhuman primates are expensive and challenging, and there are only a limited number of laboratories that have the necessary skills and resources to carry out experiments that can examine all the functions that are affected by the treatment being tested. But even though they are expensive, cumbersome and technically more complex than experiments in rodents, studies in nonhuman primates remain far less expensive than clinical trials in humans. Indeed, the financial cost associated with a single human clinical trial could support several primate studies that could lead to greater and more rapid advances in our development of treatments for SCI. The cost/benefit ratio of experiments in nonhuman primates was discussed within the framework of the current international standards for the use of experimental subjects. We recognize that experiments should be performed only if there is no other way of obtaining the results or if, as judged by the appropriate governing bodies, the benefits of the work outweigh the costs of the animals involved. On the basis of differences in the organization of sensorimotor systems between rodents and primates, as well as the safety factors that we discussed above, we argue that the limited and optimized use of nonhuman primates, such as macaque monkeys (Box 2), can be highly beneficial in efforts to improve treatment efficacy and safety in humans after SCI.

\section{Other practical considerations}

SCI models can be categorized as contusion, anatomically incomplete transection and anatomically complete transection. Each of these models has advantages and disadvantages that should be taken into account when designing translational investigations in nonhuman primates (Table 2). In any case, it is essential that the lesion model induces a permanent and reproducible deficit in at least one area of sensory, motor or autonomic function. Otherwise, the model cannot be used to test effective interventions.

Contusion. Because most human SCI results from blunt trauma, as opposed to partial or complete transection, contusion models are considered to resemble human SCI more closely than transection approaches. Contusion models can have predictable and consistent functional outcomes that allow testing of potential therapies, particularly those that mitigate the formation of lesion cavities and enhance tissue sparing.

Contusion injuries also provide a model with which to test the effect of transplanting cells or materials to fill the lesion site. However, ischemia, cavity formation and the partial tissue sparing associated with such lesions add significantly to the complexity of dissecting the mechanisms associated with functional improvements after therapeutic manipulations. When the goal is to investigate specific neural mechanisms underlying therapy-mediated recovery or to distinguish between spared and regenerated nerve fibers, transection models are preferable. Last, the sometimes severe loss of function after contusion must be weighed against the potential benefits of using this type of lesion.

Incomplete transection. Several types of incomplete spinal cord transection are suitable for testing the effects of a treatment that promotes sprouting from spared axons and, perhaps, axon regeneration. This approach is also suitable for correlating axonal changes with specific behavioral improvements. An advantage of these models is that animals rapidly recover postural control to a large extent after incomplete lesions, thereby allowing better assessment of arm and hand functions.

Another key advantage of incomplete lesions in studying motor functions is that critical physiological functions, such as bladder and bowel functions, are preserved. Also, less animal handling is needed because of partly spared postural and locomotor capabilities. Lastly, permanent, albeit modest, impairments in both fine motor control and locomotion readily allow the assessment of the potential of use-dependent mechanisms to enhance recovery ${ }^{24}$ (H. Yang et al., Soc. Neurosci. Abstr. 106.14, 2004; Wannier, T. et al., Soc. Neurosci. Abstr. 103.2, 2005).

Surgically incomplete spinal cord transections provide an avenue for investigating the 
Table 2 Advantages and disadvantages of commonly used spinal cord injury models for translational studies in nonhuman primates

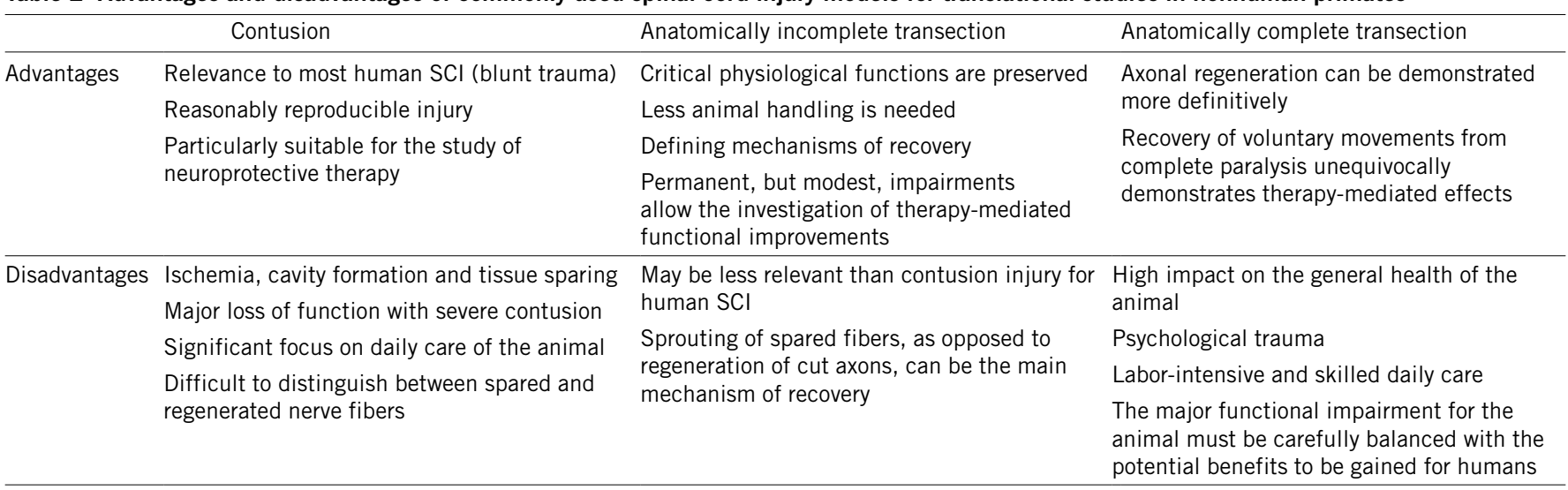

mechanisms of recovery that can be attributed to the regeneration of specific ascending or descending tracts or to intraspinal pathways. Mechanistic understanding of therapymediated motor recovery could be critical to the selection of a SCI population for a clinical trial: that is, individuals with a complete lesion would not benefit from intervention-enhanced sprouting of spared fibers, whereas such sprouting could promote significant improvements in individuals with incomplete injuries.

Incomplete spinal cord transection models have some significant advantages over contusion models and retain sufficient relevance to the human condition to be a model of choice for testing the efficacy of many therapies. However, it could become important to test the effectiveness of some therapeutic interventions after incomplete transection injuries if fundamental differences in the responses of the spinal cord to surgical and contused injuries are identified.

Complete transection. Very significant insights into the plastic mechanisms that underlie recovery of posture and locomotion after SCI have been obtained using the complete spinal cord transection model of mice, rats and cats.

So far, experiments suggest that the mechanisms of recovery from complete and incomplete SCI may be fundamentally different. Whereas true axonal regeneration may be the probable mechanism underlying functional recovery after a complete injury, local and supraspinal plasticity of intact fibers and their reorganization may be the main factor after an incomplete injury ${ }^{28}$. So, an anatomically complete transection injury could be another approach if the proposed therapy has been unequivocally shown to induce true axonal regeneration in rodents.

Nevertheless, the profound consequences of an anatomically complete transection injury on the general health of nonhuman primates, the associated psychological trauma for the animal, and the labor-intensive and skilled daily care required to maintain a desirable state of health must be carefully balanced with the potential benefit for humans. Furthermore, the possibility of obtaining the information deemed to be most critical using rodents or other nonprimate models must be thoroughly examined before consideration of using this model in nonhuman primates.

Level of lesion. The spinal level of SCIcervical, thoracic or sacral-is an important decision. Most demographic studies reveal that in humans cervical injuries are more frequent than SCI at the thoracic or lower level. Experimental lesions at the cervical level make it possible to study the recovery of manual dexterity. This paradigm affords the most detailed assessment of the recovery of fine motor control and could be a major advantage of using nonhuman primates in the translation of a therapy to humans. Even limited sprouting or regeneration in the cervical spinal cord associated with recovery of some aspects of the fine motor function can be extremely beneficial to humans.

On the other hand, injury of the nonhuman primate thoracic spinal cord allows the testing of recovery of locomotor and postural activities $^{15}$. Thoracic injuries present a different type of challenge from cervical lesions in that the descending and ascending tracts may need to form new connections over much longer distances in order to become functional.

Rehabilitation programs. Rehabilitation programs are an integral part of the care of patients after SCI. When considered in a preclinical setting, regenerative interventions for the injured nonhuman primate spinal cord should be combined with a carefully controlled rehabilitation program, as any intervention in humans will be accompanied by extensive rehabilitation. This scenario may be particularly important for regaining and maintaining precise motor control of the hands and digits.

Critical variables related to activity will need to be collected to define the interaction between use-dependent factors and the neural plasticity that can occur owing to a regenerative intervention ${ }^{29}$. Indeed, rehabilitation could drive plasticity in a useful direction, while concomitant intervention-mediated effects could be manifested poorly without training. Theoretically, training improves the function of existing circuits that can mediate coordinated movements. Nevertheless, we need to determine the optimal dosage and time point to start the regenerative intervention and rehabilitation in primates.

There is much to be gained from the study of both fine motor control and locomotion in combination with neural regenerative and usedependent factors, in terms of translating SCI studies from nonhuman primates to humans. As recovery of function is a product of neurological and use-dependent factors, nonhuman primates are a superior human surrogate for the examination of the interactions of cognitive and motivational factors associated with physical therapy and other neurobiological interventions.

\section{Conclusion}

Nonhuman primates provide unique advantages over rodents for testing and understanding the safety and efficacy of reparative interventions to promote functional recovery after SCI in humans. Nonhuman primates provide an opportunity to examine simultaneously and comprehensively the effects of a regeneration-inducing intervention on multiple variables such as fine motor control of the arm and hand, posture and locomotion (bipedal and quadrupedal), and autonomic function (such as bladder and bowel control). Capitalizing on these unique advantages is particularly significant in identifying new ways to regain control of sensorimotor 


\section{COMMENTARY}

function of distal upper limb segments and digits. Furthermore, rehabilitative therapies involving skilled manual motor tasks and bipedal locomotion could be administered to nonhuman primates on a regular basis. Finally, the high risk of a hazard and/or malfunction that could accompany an invasive therapy in human subjects underscores the advantages of understanding the safety factors in the nonhuman primate before implementing an intervention on humans.

The pathway for developing the most effective novel interventions to the greatest number of SCI patients would probably include experiments using nonhuman primates. However, most of the fundamental work on neurological diseases, including SCI, can and should continue to be performed in rodents and other animals. Nonhuman primates should be used to test invasive neural interventions that are successful in nonprimate species and, as a consequence, have a more reasonable potential for success in humans. Such studies can pinpoint the specific potential benefits, identify the mechanisms of recovery of function, and raise our confidence in the level of efficacy and safety of an intervention before we consider human clinical trials. Given the common elements underlying a range of neuromotor disorders, the potential for capitalizing on the translational studies in nonhuman primates designed to augment motor recovery following SCI extends well beyond this specific injury.

\section{ACKNOWLEDGMENTS}

This paper summarizes the discussions that took place in a workshop sponsored and organized by the Christopher Reeve Foundation (CRF). All authors are listed in alphabetical order with the exception of the primary and corresponding authors. Each author attended and took part in the discussions and in the formulation of the text. Contributions were also made by the following people: D.C. Dunbar,

P.L. Strick, C. Darian-Smith and D.A. Washburn, although they were unable to attend the workshop. We would also like to thank N. Kleitman, representative from the US National Institutes of Health, for her helpful comments. Last, we wish to thank S. Howley of the CRF for her efforts in arranging all organizational aspects of the workshop, and the CRF International Research Consortium for funding the event.

\section{COMPETING INTERESTS STATEMENT}

The authors declare no competing financial interests.

1. Anderson, K.D. J. Neurotrauma 21, 1371-1383 (2004).

2. Ackery, A., Tator, C. \& Krassioukov, A. J. Neurotrauma 21, 1355-1370 (2004).

3. Fawcett, J. Spinal Cord 40, 615-623 (2002).

4. Schwab, M.E. Curr. Opin. Neurobiol. 14, 118-124 (2004).

5. Silver, J. \& Miller, J.H. Nat. Rev. Neurosci. 5, 146-156 (2004).

6. Thuret, S., Moon, L.D. \& Gage, F.H. Nat. Rev. Neurosci. 7. 628-643 (2006).

7. Tuszynski, M.H., Grill, R., Jones, L.L., McKay, H.M. \& Blesch, A. J. Comp. Neurol. 449, 88-101 (2002).

8. Lemon, R.N., Kirkwood, P.A., Maier, M.A., Nakajima, K. \& Nathan, P. Prog. Brain Res. 143, 263-279 (2004).
9. Rouiller, E.M., Moret, V., Tanne, J. \& Boussaoud, D. Eur. J. Neurosci. 8, 1055-1059 (1996).

10. Kuypers, H.G. Prog. Brain Res. 11, 178-202 (1964).

11. Lemon, R.N. \& Griffiths, J. Muscle Nerve 32, 261-279 (2005).

12. Bortoff, G.A. \& Strick, P.L. J. Neurosci. 13, 51055118 (1993)

13. Lawrence, D.G. \& Kuypers, H.G. Brain 91, 1-14 (1968).

14. Freund, P. et al. Nat. Med. 12, 790-792 (2006).

15. Courtine, G. et al. Brain 128, 2338-2358 (2005).

16. Nathan, P.W. Brain 117, 337-346 (1994).

17. Muir, G.D. \& Whishaw, I.Q. Behav. Brain Res. 103 , 45-53 (1999).

18. Schmidlin, E., Wannier, T., Bloch, J. \& Rouiller, E.M. Brain Res. 1017, 172-183 (2004).

19. Whishaw, I.Q., Gorny, B. \& Sarna, J. Behav. Brain Res. 93, 167-183 (1998).

20. Nakajima, K. et al. Prog. Brain Res. 143, 183-190 (2004).

21. Dancause, N. et al. J. Neurosci. 25, 10167-10179 (2005).

22. Wannier, T., Schmidlin, E., Bloch, J. \& Rouiller, E.M. J. Neurotrauma 22, 703-717 (2005).

23. Schmidlin, E. et al. BMC Neurosci. 6, 56 (2005).

24. Belci, M., Catley, M., Husain, M., Frankel, H.L. \& Davey, N.J. Spinal Cord 42, 417-419 (2004).

25. Darian-Smith, C. \& Ciferri, M.M. J. Comp. Neurol. 491 27-45 (2005).

26. Hofstetter, C.P. et al. Nat. Neurosci. 8, 346-353 (2005).

27. Medical Research Council \& Wellcome Trust. Primates in Medical Research. (London, 2006).

28. Fouad, K., Klusman, I. \& Schwab, M.E. Eur. J. Neurosci. 20, 2479-2482 (2004).

29. Friel, K.M., Heddings, A.A. \& Nudo, R.J. Neurorehabil. Neural Repair 14, 187-198 (2000).

30. Heffner, R. \& Masterton, B. Brain Behav. Evol. 12 161-200 (1975).

31. Nakajima, K., Maier, M.A., Kirkwood, P.A. \& Lemon, R.N. J. Neurophysiol. 84, 698-709 (2000). 